\title{
A Matrix-Based Analytical Approach to Connection Unavailability Estimation in Shared Backup Path Protection
}

\author{
Darli A. A. Mello, Student Member, IEEE, Dominic A. Schupke, Member, IEEE, \\ and Helio Waldman, Senior Member, IEEE
}

\begin{abstract}
This letter introduces a matrix-based approach to connection unavailability estimation in shared backup path protection (SBPP). The proposed approach yields accurate results for networks of national size using simple matrix operations, being therefore suitable for online routing algorithms. The accuracy of the unavailability estimates is verified through simulations.
\end{abstract}

Index Terms-Shared Backup Path Protection (SBPP), Availability, Protection and Restoration, Matrix-Based Approach.

\section{INTRODUCTION}

In optical networks, shared backup path protection (SBPP) [1] can optimize the network resources utilization in scenarios where the connection availability requirements are not achieved without protection, but are exceeded with dedicated path protection (DPP). Previous estimates for the connection unavailability in SBPP rely on conservative upper bounds [2],[3], or approximations [4],[5], which in some cases are insufficient, e.g., if network links have different repair times.

The matrix-based approach proposed in this letter yields accurate results for practically all networks for which SBPP is of interest, while employing implementation-friendly calculations. The approach is derived from a continuous time Markov model which assumes that not more than two simultaneous link failures occur in the network. This approximation, wellaccepted in the literature, is valid for most wide-area networks.

\section{GEnERAL ASSUMPTIONS}

The derivations in this letter are based on the following assumptions:

1) A two-state "working-failed" model describes the status of all fiber links.

2) The network nodes have availability equal to one.

3) All fiber links fail independently.

4) The repair time and the time to fail of a fiber link are memoryless, exponentially distributed random processes with constant means MTTR and MTTF.

5) In SBPP, the first path to fail holds the protection resources until it is completely repaired.

Darli A. A. Mello was with the Institute of Communication Networks at the Munich University of Technology as a DAAD/FAPESP scholar, from the OptiNet/DECOM/FEEC/UNICAMP, Brazil.

Dominic A. Schupke is with Siemens AG, Corporate Technology, Information and Communications, Munich, Germany.

Helio Waldman is with OptiNet/DECOM/FEEC/UNICAMP, Campinas, Brazil.

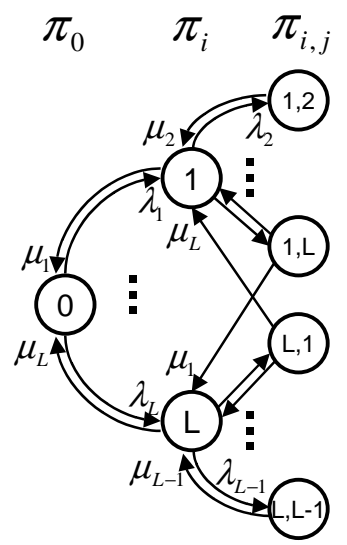

Fig. 1. The continuous time Markov chain.

6) At most two fiber links can simultaneously fail in the network.

Assumptions 1-4 are typical for transport networks. Assumption 5 is a plausible implementation of SBPP. Assumption 6 makes the approach applicable to most (even large) networks. If connections are protected against single link failures, double link failures are the dominant source of connection outages [1]. Reference [4] verifies its theoretically estimated availabilities through a double link failure simulator. For an Italian Network [6], upon the occurrence of multiple link failures, there is approximately $99 \%$ probability that only two links are in the "failed" state for a MTTR $=20 \mathrm{~h}$ and $200 \mathrm{FIT} / \mathrm{km}$ as value for $1 / \mathrm{MTTF}$ ( 1 FIT $=1$ failure in $\left.10^{9} \mathrm{~h}\right)$. In intercontinental networks this probability is lower, e.g., 95\% for the NSF Network, but in this case SBPP may be an inadequate choice to guarantee the commonly required availability levels.

\section{The MATRIX-BASED APPROACH}

The matrix-based approach consists of two steps. The first step is network specific and executed only once, during the network planning phase. The second step is connection specific and executed online, whenever the unavailability of a connection has to be assessed.

\section{A. Step I: Network Specific Calculations}

Let $L$ be the number of links of the network topology. Step I aims at obtaining matrix $\Pi_{D\left(L_{X} L\right)}$, where each element 
$\Pi_{D i, j}$ is the proportion of time during which links $i$ and $j$ are in the "failed" state, where link $i$ failed before link $j$. The elements in the main diagonal of $\boldsymbol{\Pi}_{D}$ are zeros.

The input data for Step I are the following vectors:

$\lambda$ Contains the failure rates $\lambda_{i}$ for each link $i$ of the network, where $\lambda_{i}=1 / M T T F_{i}$.

$\boldsymbol{\mu}$ Contains the repair rates $\mu_{i}$ for each link $i$ of the network, where $\mu_{i}=1 / M T T R_{i}$.

Using the assumptions of Section II the continuous time Markov chain depicted in Figure 1 can be derived. If the network is in state $(0)$, all links are operating. If the network is in state $(i)$, all links except link $i$, are operating. If the network is in state $(i, j)$, all links except links $i$ and $j$, are operating, and link $i$ failed before link $j$.

Let $\lambda_{T}$ be the sum of the failure rates of all links. The state probabilities $(\pi)$ are interrelated by the balance equations:

$$
\begin{gathered}
\left(\lambda_{T}-\lambda_{i}+\mu_{i}\right) \pi_{i}=\lambda_{i} \pi_{0}+\sum_{j=1, j \neq i}^{L} \mu_{j}\left(\pi_{i, j}+\pi_{j, i}\right), \\
\pi_{i, j}=\frac{\lambda_{j}}{\mu_{i}+\mu_{j}} \pi_{i} \\
\pi_{0}+\sum_{i=1}^{L} \pi_{i}+\sum_{i=1}^{L} \sum_{j=1, j \neq i}^{L} \pi_{i, j}=1 .
\end{gathered}
$$

Introducing Equation 2 into Equations 1 and 3 yields:

$$
\begin{aligned}
\left(\lambda_{T}-\lambda_{i}+\mu_{i}\right) \pi_{i} & =\lambda_{i} \pi_{0} \\
& +\sum_{j=1, j \neq i}^{L} \frac{\lambda_{j} \mu_{j}}{\mu_{i}+\mu_{j}} \pi_{i}+\frac{\lambda_{i} \mu_{j}}{\mu_{i}+\mu_{j}} \pi_{j} \\
\pi_{0}+\sum_{i=1}^{L} \pi_{i} & +\sum_{i=1}^{L} \sum_{j=1, j \neq i}^{L} \frac{\lambda_{j}}{\mu_{i}+\mu_{j}} \pi_{i}=1
\end{aligned}
$$

After solving Equations 4 and 5 for $\pi_{i}$, Equation 2 can be directly applied for calculating the elements $\pi_{i, j}$ of $\boldsymbol{\Pi}_{\boldsymbol{D}}$. The main advantage of using Equations 4, 5 and 2 over the regular solution procedure with the generator matrix $Q$, is a reduction in order of the matrices to be manipulated. While the regular procedure requires a matrix inversion of order $L^{2}+$ 1 , splitting the solution into two steps - first solving for $\pi_{i}$ through Equations 4 and 5 and then calculating the elements of $\Pi_{D}$ using Equation 2 - requires matrices operations of order $L+1$.

\section{B. Step II - Connection Specific Calculations}

Let $w$ be the working path of the connection whose unavailability is to be estimated, $b$ its backup path, and $s g$ the group of all working paths with backup paths that share some capacity with $b$. Consider:
$w_{\left(1_{X} L\right)}$
Contains ones in the columns correspond- ing to the links traversed by $w$, and zeros elsewhere.
$\boldsymbol{b}_{\left(\mathbf{1}_{X} \boldsymbol{L}\right)} \quad$ Contains ones in the columns corresponding to the links traversed by $b$, and zeros else- where.
$\boldsymbol{G}_{\left(S G_{X} L\right)} \quad$ Each row of $\boldsymbol{G}$ corresponds to one working path of the sharing group $s g$, and contains ones in the columns corresponding to the links traversed by the working path, and zeros elsewhere.

Step II consists in selectively adding elements of matrix $\Pi_{D}$ which represent one of the two cases:

1) The first link failure occurs in $s g$ or $b$, and the second in $w$.

2) The first link failure occurs in $w$, and the second in $b$.

Assume that the following vector and matrix operators result:
$\max (\mathbf{M})$
a row vector containing the maximum element from each column of $\mathbf{M}$.
$\operatorname{maxc}(\mathbf{v}, \mathbf{y}) \quad$ an array of the same size as $\mathbf{v}$ and $\mathbf{y}$ with the largest elements from $\mathbf{v}$ or $\mathbf{y}$.
$\operatorname{diag}(\mathbf{v})$ a square matrix with the elements of $\mathbf{v}$ in the main diagonal and zeros elsewhere.
$\operatorname{sum}(\mathbf{M})$ the sum of all elements in $\mathbf{M}$.

The connection unavailability is finally calculated as the sum of the following two terms:

$$
\begin{aligned}
\boldsymbol{U} & =\operatorname{sum}\left(\operatorname{maxc}(\max (\boldsymbol{G}), \boldsymbol{b}) \boldsymbol{\Pi}_{\boldsymbol{D}} \operatorname{diag}(\boldsymbol{w})\right) \\
& +\operatorname{sum}\left(\boldsymbol{w} \boldsymbol{\Pi}_{\boldsymbol{D}} \operatorname{diag}(\boldsymbol{b})\right) .
\end{aligned}
$$

The term $\operatorname{maxc}(\max (\boldsymbol{G}), \boldsymbol{b})$ returns a zero-one row vector representing links traversed by paths in $b$ or $s g$. Multiplying matrix $\Pi_{D}$ by the zero-one row vector on the left selects the elements of $\boldsymbol{\Pi}_{D}$ which have the links represented by the vector as first failures. Multiplying matrix $\Pi_{D}$ by a zero-one diagonal matrix on the right selects the elements of $\boldsymbol{\Pi}_{D}$ which have the links represented by the main diagonal as second failures.

The $\max$ operators in Equation 6 ensure that the contribution of links which are common to $b$ and a path in $s g$, or to more than one path in $s g$, is considered just once. The latter case does not violate the SRLG (Shared Risk Link Group) constraint if their backup paths traverse diverse links of $b$. Note that if $s g$ is empty the approach falls back to dedicated path protection.

\section{Simulation Results}

Figure 2 shows the simulation results for two scenarios in an Italian network. The network topology and link lengths can be found in [6]. Nodes were named after the three first letters of the city they represent.

Scenario one has heterogeneous MTTFs and MTTRs. This can occur, for example, in networks with submarine cables, where fiber cuts are rare and repair times are long. The routes in Table I were considered. A failure rate $=200 \mathrm{FIT} / \mathrm{km}$ and MTTR $=6 \mathrm{~h}$ were assumed in all links of the network, except 
TABLE I

Path Routes In Scenario 1

\begin{tabular}{|l|l|}
\hline$w$ & Rom-Cag-Pal \\
$b$ & Rom-Nap-Pal \\
Path 1 in $s g$ & Per-Pes-Nap-Cat-Pal \\
Path 2 in $s g$ & Anc-Pes-Bar-Pot-Catz-Cat \\
Backup of Path 1 & Per-Rom-Nap-Pal \\
Backup of Path 2 & Anc-Bolg-Fir-Rom-Nap-Pal-Cat \\
\hline
\end{tabular}

TABLE II

Path Routes In SCEnARIo 2

\begin{tabular}{|l|l|}
\hline$w$ & Fir-Rom-Nap-Pal \\
$b$ & Fir-Per-Pes-Nap-Cat-Pal \\
Path 1 in $s g$ & Bolg-Fir-Pis-Rom-Cag-Pal \\
Path 2 in sg & Fir-Pis-Rom-Cag-Pal \\
Backup of Path 1 & Bolg-Anc-Pes-Bar-Pot-Catz-Cat-Pal \\
Backup of Path 2 & Fir-Per-Pes-Nap-Pal \\
\hline
\end{tabular}

for those traversed by $w$. A failure rate $=100 \mathrm{FIT} / \mathrm{km}$ was assumed for the links traversed by $w$.

Scenario two has homogeneous MTTFs and MTTRs, but working paths in $s g$ traverse common links. This does not violate the SRLG (Shared Risk Link Group) constraint if the backup paths corresponding to the paths in $s g$ traverse diverse links of $b$. The routes in Table II were considered. A failure rate $=200 \mathrm{FIT} / \mathrm{km}$ was assumed in all network links.

Simulations were carried out by an event-based simulator which allows any possible link failure combination, of arbitrary order. The error bars in Figure 2 correspond to a confidence level of $95 \%$ on the connection unavailability in one year. We compare the results from the matrix-based approach with the estimates from the three other methods published in [4], [5], and [2]. Reference [2] assumes the conservative approximation that the connection is always unavailable if its working path $w$ and any other working path in $s g$ are in the "failed" state, independently of the order in which failures occurred. It offers therefore an upper bound on the connection unavailability.

A benefit of the matrix-based approach over previous proposals is that its scope of application is delineated by the network size, and not by the routing of connections in the network. Since it assumes that at most two fiber links can simultaneously fail in the network, it yields a tight lower bound on the connection unavailability. A quantitative measure for the tightness of the results is the probability that only two link failures are in the "failed" state, given that the network contains multiple link failures. This is an initially checkable property of the network, and is therefore independent of the network occupation. For the simulated Italian network it is approximately $99 \%$ for a MTTR $=20 \mathrm{~h}$ and a link failure rate of $200 \mathrm{FIT} / \mathrm{km}$, which guarantees that for this network the matrix-based approach offers accurate estimates. For networks of national size, given the dual failure dominance, the approach yields accurate estimates with negligible error.
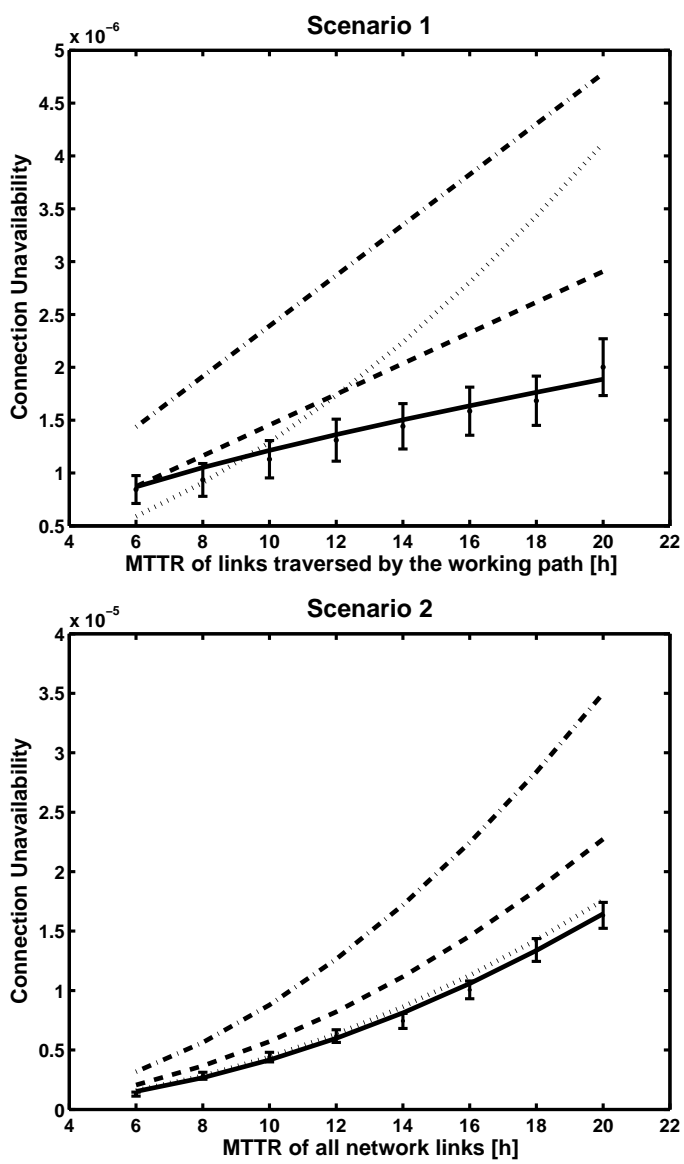

Fig. 2. Unavailability estimates for two scenarios. Dotted line: calculation method of [4]. Dashed line: calculation method of [5]. Dash-dotted line: calculation method of [2]. Solid line: matrix-based approach. The dots with confidence intervals (at 95\% confidence level) were obtained by simulations.

\section{CONCLUSION}

The matrix-based approach is an efficient method for connection unavailability estimation in shared backup path protection. In networks of national dimensions, for which this protection scheme is particularly attractive, the approach yields accurate results for arbitrary link repair and failure rate parameters. Finally, its low computation complexity makes it suitable for online and management-based routing.

\section{REFERENCES}

[1] J. Doucette, M. Clouqueur, and W. Grover, "On the availability and capacity requirements of shared backup path-protected mesh networks," Optical Networks Magazine, vol. 4, no. 6, pp. 29-44, Nov./Dec. 2003.

[2] D. Arci, D. Petecchi, G. Maier, A. Pattavina, and M. Tornatore, "Availability models for protection techniques in WDM networks," in Proc. DRCN'03, Banff, Canada, Oct. 2003, pp. 158-166.

[3] M. Tacca, A. Fumagalli, and F. Unghvary, "Double-fault shared path protection scheme with constrained connection downtime," in Proc. DRCN'03, Banff, Canada, Oct. 2003, pp. 181-188.

[4] M. Jaeger and R. Huelsermann, "Service availability of shared path protection in optical mesh networks," in Proc. ECOC'04, Stockholm, Sweden, Sept. 2004.

[5] J. Zhang, K. Zhu, H. Zang, and B. Mukherjee, "A new provisioning framework to provide availability-guaranteed service in WDM mesh networks," in Proc. ICC'03, Anchorage, USA, May 2003, pp. 1484-1488.

[6] M. Ali and S. Deogun, "Power-efficient design of multicast wavelengthrouted networks," IEEE J. Select. Areas Commun., vol. 18, pp. 1852 1862 , Oct. 2000. 